clinical care and deliver improved end-of-life care in the community.

\section{P-232 ENRICHING AND UPSKILLING HEALTH CARE PROFESSIONALS THROUGH VIRTUAL LEARNING DURING COVID-19}

Angela Cooke. ellenor Hospice, Northfleet, UK

10.1136/spcare-2021-Hospice.246

Background In April 2020, the education department at a hospice recognised that the COVID-19 pandemic had highlighted the importance of enhancing the education on end-of-life care for care home and community care staff. Hence, teaching sessions aimed at health care professionals (HCP) within the hospice's catchment area were arranged.

Aim To support HCPs to build confidence in caring for patients dying in their place of care, and to avoid unnecessary hospital admissions.

Methods Community care providers and the local authority were asked to promote the training sessions that were based on several palliative care topics. These hourly sessions were delivered by a registered nurse who was experienced in delivering training in palliative care, together with assistance from the hospice's physiotherapist and head of wellbeing. All sessions were delivered virtually and were offered during the day and the evening.

Results Due to the high uptake of the initial sessions, a collaborative was subsequently formed with two other hospices and an NHS trust to support the delivery of this training. Topics included: Bereavement, Understanding Breathlessness, General Lung Health, Energy Conservation, Symptom Assessment and Management, Drug Calculations, Remote Assistance for Verification of Expected Death (VOED) by Health Care Assistants, VOED by Registered Nurses, Recognising Dying and Care of the Dying Patient. Over the course of 12 months and 128 sessions, 2,751 individuals from the UK, Canada, Australia and Singapore attended. These attendees were from a mix of 22 community care providers and acute settings. The attendees highlighted that the evening sessions were beneficial due to an increased workload during the day.

Conclusion By utilising virtual platforms, the success of this training programme exceeded the initial expectations, and the training was well attended by learners worldwide. This newly formed collaborative group will continue to deliver palliative care training collectively. 